\title{
Dysfonction érectile et traitement antihypertenseur
}

\author{
Dominique STEPHAN, Sébastien GAERTNER, Cécile BERTRAND, Julie QUELET
}

Service hypertension artérielle et maladies vasculaires, pharmacologie clinique, CHRU Strasbourg

\section{RÉSUMÉ}

Une dysfonction érectile préexiste chez 15 à $20 \%$ des patients hypertendus avant même l'introduction d'un traitement antihypertenseur. La fréquence de la dysfonction érectile passe à $\mathbf{4 0}$ - $\mathbf{5 0} \%$ sous traitement antihypertenseur. Les études cliniques de bonne méthodologie ont montré que les diurétiques étaient les principaux pourvoyeurs de dysfonction érectile. La plurithérapie antihypertensive est un facteur aggravant. Chez l'hypertendu souffrant d'insuffisance érectile, les inhibiteurs des phosphodiestérases 5 peuvent être utilisés après exclusion des contre-indications.

Mots clés : dysfonction érectile, antihypertenseurs, inhibiteurs des phosphodiestérases 5

\section{INTRODUCTION}

La dysfonction érectile est une plainte fréquente chez les hypertendus lors du suivi cardiovasculaire. Il peut s'agir de patients nouvellement diagnostiqués pour leur hypertension artérielle (HTA) et chez lesquels la décision est prise d'instituer un traitement antihypertenseur. Dans les notices d'information concernant certains médicaments, il est clairement indiqué que le médicament est susceptible de provoquer une impuissance. C'est notamment le cas des diurétiques, des bêta-bloquants ou des antihypertenseurs centraux. Dans ce cas, le patient peut craindre dès la lecture de la notice d'information qu'il va souffrir d'une insuffisance érectile.

Dans d'autres cas, il s'agit de patients jusque là contrôlés par un traitement antihypertenseur comprenant souvent un bêta-bloquant qui évoque le problème de l'impuissance au cours d'une consultation. C'est parfois une rencontre et la reprise d'une activité sexuelle qui amènent le patient à demander auprès de son prescripteur un changement thérapeutique. Dans d'autres cas, le symptôme d'insuffisance érectile est caché derrière une plainte d'une autre nature telle qu'une fatigue chronique, un manque de motivation ou une sensation de jambes coupées.

Dans le dictionnaire VIDAL, le résumé des caractéristiques pharmacologiques de plus d'une cinquantaine de molécules mentionne la possibilité d'une impuissance. II s'agit en premier lieu des bêta-bloquants mais également des antihypertenseurs centraux, des alpha-bloquants et des diurétiques qu'il s'agissent des diurétiques thiazidiques ou anti-aldostérone. II y a entre neuf à dix millions d'hypertendus en France, c'est donc pour le praticien un problème thérapeutique fréquent.

\section{INSUFFISANCE ÉRECTILE ET TRAITEMENT ANTI-HYPERTENSEUR}

La célèbre Massachusetts Male Aging Study (MMAS) du groupe de Boston a montré que la dysfonction érectile était plus fréquente chez les sujets qui présentent des facteurs de risque cardiovasculaires associés [5]. Ainsi l'hypertension artérielle (HTA) ou l'administration d'un traitement antihypertenseur double le risque relatif de dysfonction érectile. Pour rappel, ce risque est triplé chez l'homme de plus de 60 ans, multiplié par 2 chez le tabagique et par 1,5 en cas de surcharge pondérale. La fréquence de l'HTA augmentant avec l'âge, on comprend que l'addition de ces deux facteurs de risque contribue à majorer le risque d'impuissance chez l'hypertendu.

Une étude épidémiologique plus récente précise la fréquence de la dysfonction érectile en fonction de la présence des

\section{Correspondance :}

Pr Dominique STEPHAN - Service hypertension artérielle et maladies vasculaires, 1 place de l'Hôpital, 67091 BP 426 Strasbourg cedex - Tel 0388116176 - Fax 0388116431 -

Email dominique.stephan@chru-strasbourg.fr 
facteurs de risque cardiovasculaire et des maladies cardiovasculaires associées [8]. Ainsi une dysfonction érectile est retrouvée chez $35,4 \%$ des patients hypertendus. Cette prévalence atteint $52 \%$ pour les cardiopathies, $50 \%$ pour le diabète, $23,5 \%$ en cas d'obésité et $15 \%$ chez les patients tabagiques. Rappelons qu'environ $70 \%$ des diabétiques sont hypertendus pour évaluer l'importance potentielle du problème de l'impuissance masculine.

En France, l'étude du Groupe des Jeunes Hypertensiologues a mesuré la fréquence des troubles de la sexualité chez des hommes et des femmes hypertendus avant et après l'introduction d'un traitement antihypertenseur [4]. II s'agissait d'une enquête nationale multicentrique faisant appel à un auto-questionnaire pratiqué chez 459 hypertendus. Cette étude a montré l'existence de troubles de la sexualité avant le traitement chez 42 sur 248 hommes (17\%) et 18 sur 142 femmes $(13 \%)$. La fréquence des troubles de la sexualité a ensuite été mesurée après l'introduction du traitement antihypertenseur. Chez les hommes, on note dans $49 \%$ des cas, des modifications de la sexualité sous traitement. $\mathrm{Ce}$ chiffre est de $18 \%$ chez les femmes. Chez $45 \%$ des hommes, on note des troubles de l'érection, les érections sous traitement étant moins fréquentes dans $31 \%$ des cas, moins durables dans $19 \%$ et impossibles dans $11 \%$ des cas. Dans cette étude, les principaux facteurs de risque de trouble de la sexualité chez l'hypertendu était la polythérapie antihypertensive ( $p<0,01)$, la coexistence d'une coronaropathie $(p=0,01)$ et un traitement diurétique $(p=$ 0,03 ).

Les effets des traitements antihypertenseurs sur la sexualité des patients hypertendus ont été étudiés dans d'autres essais randomisés, le plus souvent pratiqués en double insu et comparant, pour les plus anciens, bêta-bloquants et diurétiques au placebo et pour les essais plus récents, inhibiteurs de l'enzyme de conversion, inhibiteurs calciques aux molécules plus anciennes. Les résultats de ces essais montrent que les difficultés d'érection sont significativement plus importantes chez les patients traités par diurétiques. En moyenne, $23 \%$ des patients recevant une molécule diurétique ont une insuffisance érectile.

L'étude TOMHS a comparé la fréquence des difficultés d'érection chez les patients recevant un bêta-bloquant, un diurétique, un inhibiteur de l'enzyme de conversion ou un inhibiteur calcique par rapport au placebo [3]. Dans cette étude, les diurétiques augmentaient significativement la fréquence des dysfonctions érectiles. II n'y avait pas de différences significatives entre le groupe placebo et le groupe bêta-bloquant, le groupe inhibiteur de l'enzyme de conversion ou le groupe inhibiteur calcique.

II reste que les bêta-bloquants sont fréquemment associés à la crainte d'une insuffisance érectile et ce rôle péjoratif pourrait résulter de la simple lecture de la notice d'information du médicament le mentionnant. Néanmoins, l'avènement de molécules bêta-bloquantes disposant d'activités vasodilatatrices périphériques pourrait réduire la fréquence des dysfonctions érectiles chez l'hypertendu. Ainsi le nébivolol, molécule bêta-bloquante cardiosélective augmentant la production de monoxyde d'azote par l'endothélium a montré sa capacité à induire moins d'insuffisance érectile que les bêta-bloquants traditionnels (aténolol) ou les molécules très cardiosélectives (métoprolol et bisoprolol) [2]. Cette activité bénéfique du nébivolol pourrait être directement liée à sa capacité à majorer l'élaboration de monoxyde d'azote par l'endothélium car on sait que la dysfonction érectile résulte en partie d'une dysfonction endothéliale [7].

\section{INHIBITEUR DES PHOSPHODIESTÉRASES 5 ET TRAITEMENT ANTIHYPERTENSEUR}

Le sildénafil (Viagra®) a été étudié dans des essais cliniques rassemblant plus de 3000 patients âgés de 19 à 87 ans. Dans $24 \%$ des cas il s'agissait de patients porteurs d'une hypertension artérielle. Le résumé de ces essais montre que le sildénafil est capable d'induire une baisse de la pression artérielle diastolique d'environ $8 \mathrm{mmHg}$ et de la pression systolique d'environ $5 \mathrm{mmHg}$. Cependant, ces essais ne mentionnent pas de conséquences cardiovasculaires défavorables lors de la co-prescription avec les diurétiques, les inhibiteurs calciques, les inhibiteurs de l'enzyme de conversion, les alpha ou bêta-bloquants.

Les contre-indications cardiovasculaires des inhibiteurs de la phosphodiestérase de type 5 (iPDE5) doivent cependant être respectées chez l'hypertendu. II est ainsi contre-indiqué d'utiliser un IPDE5 dans les situations suivantes : prise de dérivés nitrés dans les 24 à 48 heures précédant la prise d'un IPDE5 ; prise simultanée de donneurs de monoxyde d'azote (molsidomine ou nicorandil) ; infarctus du myocarde récent (moins de six mois) ; hypotension artérielle $<90 / 50 \mathrm{mmHg}$ ; rétrécissement aortique calcifié serré ou de cardiomyopathie obstructive [6].

\section{CONCLUSION}

Devant un trouble sexuel survenant sous antihypertenseur, il faut s'enquérir de la situation avant l'introduction du traitement antihypertenseur. En effet des difficultés d'érection à divers degrés peuvent préexister chez le patient souffrant d'hypertension artérielle. II faut ensuite mesurer l'impact du traitement antihypertenseur sur la fonction érectile. L'utilisation des nouvelles classes médicamenteuses recommandées dans le traitement de l'hypertension artérielle et qui privilégie les inhibiteurs du système rénine angiotensine (IEC - sartans) et les inhibiteurs calciques devrait limiter la fréquence de la survenue de cet effet indésirable.

Chez un patient chez lequel l'utilisation des bêtabloquants est formellement indiquée, le choix du nébivolol qui augmente la production de monoxyde d'azote par l'endothélium peut s'avérer judicieux. Si le bêta-bloquant n'est pas formellement indiqué, il est possible de changer de classe thérapeutique. Dans d'autres cas, il faudra garder le traitement et traiter l'impuissance en utilisant les inhibiteurs de phosphodiestérase 5 [1].

Dans tous les cas une information claire quant aux effets indésirables des médicaments se révèle un message indispensable à la qualité de l'échange entre médecins et patients. 


\section{RÉFÉRENCES}

1. DOUMAS M., DOUMA S. : The effect of antihypertensive drugs on erectile dysfunction : a proposed management algorithm. J. Clin. Hypertens., 2006, 8 : 359-364.

2. DOUMAS M., TSAKIRIS A., DOUMAS. et al. : Beneficial effects of switching from b-Blockers to nebivolol on the erectile function of hypertensive patients. Asian J. Androl., 2006, $8:$ 177-182.

3. GRIMM R.H., GRANDITS G.A., PRINEAS R.J. et al. : Long term effects on sexual function of five antihypertensive drugs and nutritional hygienic treatment in hypertensive men and women (TOHMS). Hypertension, 1997, $29: 8-14$.

4. HANON O., MOUNIER-VEHIER C., FAUVEL J.P. et al. : Troubles de la sexualité chez les hypertendus traités. Arch. Mal. Cœur, 2002, 95 : 673-677.

5. JOHANNES C.B., ARAUJO A.B., FELDMAN H.A., DERBY C.A., KLEINMAN K.P., MCKINLEY J.B. : Incidence of erectile dysfunction in men $\mathbf{4 0}$ to 69 years old : longitudinal results from the Massachusetts male aging study. J. Urol., 2000, $163: 460-463$.

6. PHILIPPE F., BONDIL P. : La dysfonction érectile un nouveau symptôme pour le cardiologue. Ann. Angeiol. Cardiol., 2006, 55 : 187-191.

7. SOLOMON H., MAN J.W., JACKSON G. : Erectile dysfunction and the cardiovascular patient : endothelial function is the common denominator. Heart, 2003, $89: 251$.

8. THOMPSON I.M., TANGEN C.M., GOODMAN P.J. et al. : Erectile dysfunction and subsequent cardiovascular disease. J. Am. Med. Ass., 2005, 294 : 2996-3002.

Manuscrit reçu : janvier 2008 ; accepté février 2008.

Communication présentée lors du XXIVème Congrès de la SALF, Colmar décembre 2007.

\section{ABSTRACT}

\section{ERECTILE DYSFUNCTION AND ANTIHYPERTENSIVE DRUGS}

\author{
Dominique STEPHAN, Sébastien GAERTNER, \\ Cécile BERTRAND, Julie QUELET
}

Fifteen to $20 \%$ of hypertensive patients suffer from erectile dysfunction prior to initiation of antihypertensive therapy and the frequency of erectile dysfunction increases to $40-50 \%$ after initiation of antihypertensive therapy.

Clinical studies conducted according to a rigorous methodology have shown that diuretics are the main cause of erectile dysfunction. Antihypertensive combination therapy is an aggravating factor.

Phosphodiesterase-5 inhibitors can be used in hypertensive patients with erectile insufficiency in the absence of any contraindications.

Key Words: erectile dysfunction, antihypertensive drugs, phosphodiesterase inhibitors 